\title{
Synthesis of Novel Goniothalamin Analogs
}

\author{
Rosimeire C. Barcelos and Ronaldo A. Pilli* \\ Instituto de Química, Unicamp, Campinas, São Paulo, Brasil \\ *pilli@iqm.unicamp.br
}

Keywords: goniothalamin, biological activity, lactone analogs.

\section{INTRODUCTION}

The styryl lactone goniothalamin (1) is a secondary metabolite distributed among the plants of the genus Goniothalamus which has cytotoxic and antiproliferative properties. ${ }^{1,2}$

Given the interest of our research group to pursue studies of the biological activity of compounds based on its structure, we proposed the synthesis of hydrophilic analogs (2a-c).

Moreover, the incorporation of fluorine into molecules has become a common tool in medicinal chemistry for improving the pharmacological profile of bioactive compounds. ${ }^{3-5}$ Thus, we proposed the synthesis of compounds (2d-e) containing the trifluoromethyl groups.

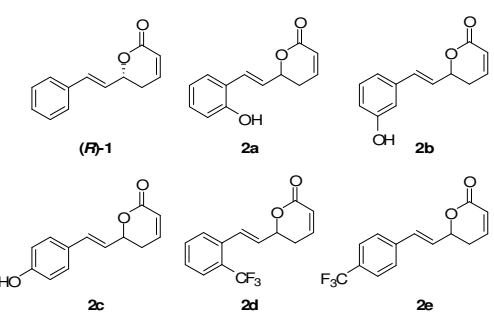

Figure 1. $(R)$-goniothalamin (1) and analogs (2a-e).

\section{RESULTS AND DISCUSSION}

The general synthetic route to achieve analogs 2a-e is represented in Scheme 1.
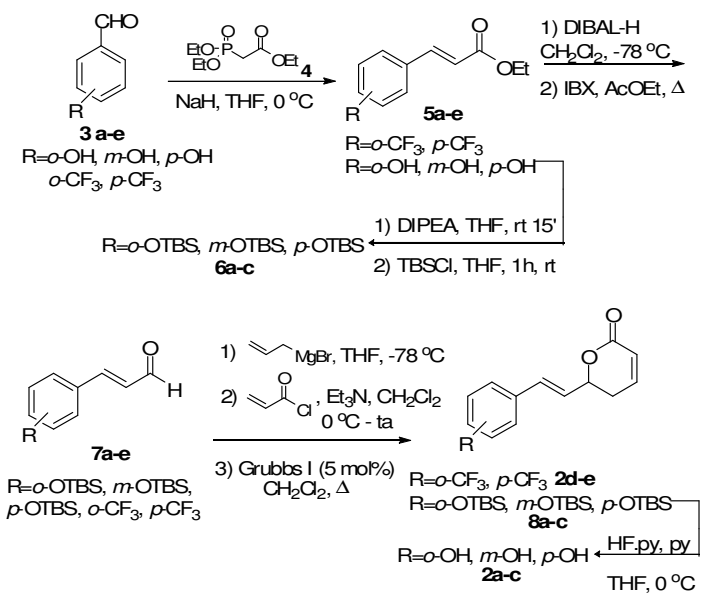

Scheme 1. General synthetic route for goniothalamin analogs 2a-e.
The synthesis of lactones 2a-e began with the preparation of aldehydes 7a-e. Reagent 4 was used to homologate commercial aldehydes 3a-e by the HWE reaction, followed by reduction of esters 5a-e with DIBAL-H and oxidation with IBX, leading to the corresponding aldehydes 7a-e. The hydroxyl groups were protected using TBSCl and DIPEA.

Allyl Grignard was added to generate the homoallylic alcohols which were reacted with acryloyl chloride leading to the corresponding esters. Finally, the lactone analogs were achieved by treating the dienes with Grubbs I catalyst (5 mol\%). The TBS groups were removed using solution of HF in pyridine. The overall yields are summarized in Table 1.

Table 1. Overall yields for goniothalamin analogs 2a-e.

\begin{tabular}{|c|c|c|}
\hline Analogs & $\mathbf{R}$ & Overall yields \\
\hline $2 a$ & $\mathrm{O}-\mathrm{OH}$ & $22 \%$ * \\
\hline $2 b$ & $m-\mathrm{OH}$ & $38 \%{ }^{*}$ \\
\hline 2c & $p-\mathrm{OH}$ & $17 \%$ * \\
\hline $2 d$ & $o-\mathrm{CF}_{3}$ & $15 \%$ ** \\
\hline $2 e$ & $p-\mathrm{CF}_{3}$ & $17 \%$ ** \\
\hline
\end{tabular}

\section{CONCLUSION}

The present work discloses the synthesis of five novel lactone analogs (2a-e) of goniothalamin. These compounds will be evaluated in vitro against human tumor cell lines in an antiproliferative assay and also for the inhibition of protein phosphatase.

\section{ACKNOWLEDGEMENTS}

The authors thank FAPESP, CAPES and CNPq for financial support.

\section{REFERENCES}

${ }^{1}$ Vendramini-Costa, D. B.; de Castro, I. B. D.; Ruiz, A. L. T. G. Marquissolo, C. Pilli, R. A.; de Carvalho, J. E. Bioorg. Med. Chem. 2010 18, 6742. ${ }^{2}$ de Fátima, A.; Zambuzzi, W. F.; Modolo, L. V.; Tarsitano, C. A B.; Gadelha, F. R.; Hyslop, S.; de Carvalho, J. E.; Salgado, I.; Ferreira, C. V.; Pilli, R. A. Chem. Biol. Interact. 2008, 176, 143. ${ }^{3}$ Zhang, S.; Zhang, Y.; Ji, Y.; Li, H.; Wang, W. Chem. Commun. 2009, 4886. 'Hao, L.; Yafei, L.; Shilei, Z.; ChenGuang, Y.; Wei, W. Science China Chemistry. 2010, 53, 135. ${ }^{5}$ Dumitrescu, L.; Huong, D. T. M.; Hung, N. V.; Crousse, B. European Journal of Medicinal Chemistry. 2010, 45, 3213. 